\title{
MAJORIZATION INEQUALITIES VIA PEANO'S REPRESENTATION OF HERMITE'S POLYNOMIAL
}

\author{
N. LATIF ${ }^{1, *}$, N. SIDDIQUE ${ }^{2}$ AND J. PEČARIĆ ${ }^{3,4}$ \\ ${ }_{1, *}$ Department of General Studies, Jubail Industrial College, Jubail Industrial City 31961, Kingdom of \\ Saudi Arabia \\ ${ }^{2}$ Department of Mathematics, Govt. College University, Faisalabad 38000, Pakistan \\ ${ }^{3}$ Faculty of Textile Technology Zagreb, University of Zagreb, Prilaz Baruna Filipovića 28A, 10000 Zagreb, \\ Croatia \\ ${ }^{4}$ RUDN University, 6 Miklukho-Maklay St, Moscow, 117198, Russia \\ *Corresponding author: naveed707@gmail.com
}

\begin{abstract}
The Peano's representation of Hermite polynomial and new Green functions are used to construct the identities related to the generalization of majorization type inequalities in discrete as well as continuous case. $\check{C}$ ebyšev functional is used to find the bounds for new generalized identities and to develop the Grüss and Ostrowski type inequalities. Further more, we present exponential convexity together with Cauchy means for linear functionals associated with the obtained inequalities and give some applications.
\end{abstract}

Received 2018-01-05; accepted 2018-02-20; published 2018-05-02.

2010 Mathematics Subject Classification. 26D07, 26D15, 26D20, 26 D99.

Key words and phrases. classical majorization theorem; Fuchs's thorem; Peano's representation of Hermite's polynomial; Green function for 'two point right focal' problem; Čebyšev functional; Grüss type upper bounds; Ostrowski-type bounds; n-exponentially convex function; mean value theorems; Stolarsky type means.

(C) 2018 Authors retain the copyrights of their papers, and all open access articles are distributed under the terms of the Creative Commons Attribution License. 


\section{Introduction ANd Preliminaries}

Newton and Lagrange gave the classical methods for constructing Hermite interpolating polynomial. Lagrange gave the method for such function $f(t)$ is defined at the distinct increasing points $a_{1}, a_{2}, \ldots, a_{n}$ but Newton gave the method for such function $f(t)$ is defined at the distinct (but not necessarily increasing) points $a_{1}, a_{2}, \ldots, a_{n}$ (see $\left.[3,7]\right)$.

We start with a brief overview of divided differences and $n$-convex functions and give some basic results from the majorization theory.

An $n$th order divided difference of a function $\phi:[\alpha, \beta] \rightarrow \mathbb{R}$ at distinct points $x_{0}, x_{1}, \ldots, x_{n} \in[\alpha, \beta]$ may be defined recursively by

$$
\begin{aligned}
{\left[x_{i} ; \phi\right] } & =\phi\left(x_{i}\right), \quad i=0, \ldots, n, \\
{\left[x_{0}, \ldots, x_{n} ; \phi\right] } & =\frac{\left[x_{1}, \ldots, x_{n} ; \phi\right]-\left[x_{0}, \ldots, x_{n-1} ; \phi\right]}{x_{n}-x_{0}} .
\end{aligned}
$$

The value $\left[x_{0}, \ldots, x_{n} ; \phi\right]$ is independent of the order of the points $x_{0}, \ldots, x_{n}$.

A function $\phi$ is $n$-convex on $[\alpha, \beta]$ if

$$
\left[x_{0}, x_{1}, \ldots, x_{n} ; \phi\right] \geq 0
$$

holds for all choices of $(n+1)$ distinct points $x_{i} \in[\alpha, \beta], i=0, \ldots, n$.

Remark 1.1. From this definition it follows that 1-convex function is increasing function and 2-convex function is just convex function. If $\phi^{(n)}$ exists, then $\phi$ is $n$-convex iff $\phi^{(n)} \geq 0$. Also, if $\phi$ is $n$-convex for $n \geq 2$, then $\phi^{(k)}$ exists and $\phi$ is $(n-k)$-convex for $1 \leq k \leq n-2$. For more informations see [13].

On the basis of various applications of the divided differences, several representations have been obtained like error representation, Cauchy's representation, Newton's representation and Peano's representation. In this paper, we give the generalized results with the connection of Peano's representation of Hermite's interpolating polynomial and newly defined Green functions.

Majorization makes precise the vague notion that the components of a vector $\mathbf{y}$ are "less spread out" or "more nearly equal" than the components of a vector $\mathbf{x}$. A complete and superb reference on the subject is the 2011 book by Marshall et al. [12].

For fixed $m \geq 2$ let

$$
\mathbf{x}=\left(x_{1}, \ldots, x_{m}\right), \mathbf{y}=\left(y_{1}, \ldots, y_{m}\right)
$$

denote two real $m$-tuples. Let

$$
\begin{gathered}
x_{[1]} \geq x_{[2]} \geq \ldots \geq x_{[m]}, \quad y_{[1]} \geq y_{[2]} \geq \ldots \geq y_{[m]}, \\
x_{(1)} \leq x_{(2)} \leq \ldots \leq x_{(m)}, \quad y_{(1)} \leq y_{(2)} \leq \ldots \leq y_{(m)}
\end{gathered}
$$

be their ordered components. 
Definition 1.1. [13, p. 319] $\boldsymbol{x}$ is said to majorize $\boldsymbol{y}$ (or $\boldsymbol{y}$ is said to be majorized by $\boldsymbol{x}$ ), in symbol, $\boldsymbol{x} \succ \boldsymbol{y}$, if

$$
\sum_{i=1}^{l} y_{[i]} \leq \sum_{i=1}^{l} x_{[i]}
$$

holds for $l=1,2, \ldots, m-1$ and

$$
\sum_{i=1}^{m} x_{i}=\sum_{i=1}^{m} y_{i}
$$

Note that (1.1) is equivalent to

$$
\sum_{i=m-l+1}^{m} y_{(i)} \leq \sum_{i=m-l+1}^{m} x_{(i)}
$$

holds for $l=1,2, \ldots, m-1$.

The following theorem is well-known as the majorization theorem given by Marshall et al. [12, p. 14] (see also [13, p. 320]):

Theorem 1.1. Let $\boldsymbol{x}=\left(x_{1}, \ldots, x_{m}\right), \boldsymbol{y}=\left(y_{1}, \ldots, y_{m}\right)$ be two $m$-tuples such that $x_{i}, y_{i} \in[\alpha, \beta](i=1, \ldots, m)$. Then

$$
\sum_{i=1}^{m} f\left(y_{i}\right) \leq \sum_{i=1}^{m} f\left(x_{i}\right)
$$

holds for every continuous convex function $f:[\alpha, \beta] \rightarrow \mathbb{R}$ if and only if $\mathbf{x} \succ \mathbf{y}$ holds.

The following theorem can be regarded as a weighted version of Theorem 1.1 and is proved by Fuchs in [8] $([12$, p. 580], [13, p. 323]):

Theorem 1.2. Let $\boldsymbol{x}=\left(x_{1}, \ldots, x_{m}\right), \boldsymbol{y}=\left(y_{1}, \ldots, y_{m}\right)$ be two decreasing real m-tuples with $x_{i}, y_{i} \in[\alpha, \beta]$ $(i=1, \ldots, m)$ and $\mathbf{w}=\left(w_{1}, w_{2}, \ldots, w_{m}\right)$ be a real $m$-tuple such that

$$
\sum_{i=1}^{l} w_{i} y_{i} \leq \sum_{i=1}^{l} w_{i} x_{i} \text { for } l=1, \ldots, m-1,
$$

and

$$
\sum_{i=1}^{m} w_{i} y_{i}=\sum_{i=1}^{m} w_{i} x_{i}
$$

Then for every continuous convex function $f:[\alpha, \beta] \rightarrow \mathbb{R}$, we have

$$
\sum_{i=1}^{m} w_{i} f\left(y_{i}\right) \leq \sum_{i=1}^{m} w_{i} f\left(x_{i}\right) .
$$

The following integral version of Theorem 1.2 is a simple consequence of Theorem 12.14 in [15] (see also [13, p.328]): 
Theorem 1.3. Let $x, y:[a, b] \rightarrow[\alpha, \beta]$ be decreasing and $w:[a, b] \rightarrow \mathbb{R}$ be continuous functions. If

$$
\int_{a}^{\nu} w(t) y(t) d t \leq \int_{a}^{\nu} w(t) x(t) d t \quad \text { for every } \nu \in[a, b]
$$

and

$$
\int_{a}^{b} w(t) y(t) d t=\int_{a}^{b} w(t) x(t) d t
$$

hold, then for every continuous convex function $f:[\alpha, \beta] \rightarrow \mathbb{R}$, we have

$$
\int_{a}^{b} w(t) f(y(t)) d t \leq \int_{a}^{b} w(t) f(x(t)) d t
$$

Let $-\infty<\alpha<\beta<\infty$ and $\alpha \leq a_{1}<a_{2} \cdots<a_{r} \leq \beta,(r \geq 2)$ be the given points. For $f \in C^{n}[\alpha, \beta]$ a unique polynomial $\rho_{H}(s)$ of degree $(n-1)$ exists satisfying any of the following conditions:

\section{Hermite conditions:}

$$
\rho_{H}^{(i)}\left(a_{j}\right)=f^{(i)}\left(a_{j}\right) ; 0 \leq i \leq k_{j}, 1 \leq j \leq r, \sum_{j=1}^{r} k_{j}+r=n .
$$

It is of great interest to note that Hermite conditions include the following particular cases:

Type $(m, n-m)$ conditions: $\left(r=2,1 \leq m \leq n-1, k_{1}=m-1, k_{2}=n-m-1\right)$

$$
\begin{gathered}
\rho_{(m, n)}^{(i)}(\alpha)=f^{(i)}(\alpha), 0 \leq i \leq m-1, \\
\rho_{(m, n)}^{(i)}(\beta)=f^{(i)}(\beta), 0 \leq i \leq n-m-1,
\end{gathered}
$$

Two-point Taylor conditions: $\left(n=2 m, r=2, k_{1}=k_{2}=m-1\right)$

$$
\rho_{2 T}^{(i)}(\alpha)=f^{(i)}(\alpha), \rho_{2 T}^{(i)}(\beta)=f^{(i)}(\beta), 0 \leq i \leq m-1
$$

We have the following result from [3].

Theorem 1.4. Let $-\infty<\alpha<\beta<\infty$ and $\alpha \leq a_{1}<a_{2} \cdots<a_{r} \leq \beta,(r \geq 2)$ be the given points, and $f \in C^{n}([\alpha, \beta])$. Then we have

$$
f(t)=\rho_{H}(t)+R_{H, n}(f, t)
$$

where $\rho_{H}(t)$ is the Hermite interpolating polynomial, i.e.

$$
\rho_{H}(t)=\sum_{j=1}^{r} \sum_{i=0}^{k_{j}} H_{i j}(t) f^{(i)}\left(a_{j}\right)
$$

the $H_{i j}$ are fundamental polynomials of the Hermite basis defined by

$$
H_{i j}(t)=\left.\frac{1}{i !} \frac{\omega(t)}{\left(t-a_{j}\right)^{k_{j}+1-i}} \sum_{k=0}^{k_{j}-i} \frac{1}{k !} \frac{d^{k}}{d t^{k}}\left(\frac{\left(t-a_{j}\right)^{k_{j}+1}}{\omega(t)}\right)\right|_{t=a_{j}}\left(t-a_{j}\right)^{k}
$$




$$
\omega(t)=\prod_{j=1}^{r}\left(t-a_{j}\right)^{k_{j}+1}
$$

and the remainder is given by

$$
R_{H, n}(f, t)=\int_{\alpha}^{\beta} G_{H, n}(t, s) f^{(n)}(s) d s
$$

where $G_{H, n}(t, s)$ is defined by

$$
G_{H, n}(t, s)=\left\{\begin{array}{l}
\sum_{j=1}^{l} \sum_{i=0}^{k_{j}} \frac{\left(a_{j}-s\right)^{n-i-1}}{(n-i-1) !} H_{i j}(t) ; s \leq t \\
-\sum_{j=l+1}^{r} \sum_{i=0}^{k_{j}} \frac{\left(a_{j}-s\right)^{n-i-1}}{(n-i-1) !} H_{i j}(t) ; s \geq t
\end{array}\right.
$$

for all $a_{l} \leq s \leq a_{l+1} ; l=0, \ldots, r$ with $a_{0}=\alpha$ and $a_{r+1}=\beta$.

Remark 1.2. In particular cases,

for type $(m, n-m)$ conditions, from Theorem 1.4 we have

$$
f(t)=\rho_{(m, n)}(t)+R_{(m, n)}(f, t)
$$

where $\rho_{(m, n)}(t)$ is $(m, n-m)$ interpolating polynomial, i.e

$$
\rho_{(m, n)}(t)=\sum_{i=0}^{m-1} \tau_{i}(t) f^{i}(\alpha)+\sum_{i=0}^{n-m-1} \eta_{i}(t) f^{i}(\beta)
$$

with

$$
\tau_{i}(t)=\frac{1}{i !}(t-\alpha)^{i}\left(\frac{t-\beta}{\alpha-\beta}\right)^{n-m} \sum_{k=0}^{m-1-i}\left(\begin{array}{c}
n-m+k-1 \\
k
\end{array}\right)\left(\frac{t-\alpha}{\beta-\alpha}\right)^{k}
$$

and

$$
\eta_{i}(t)=\frac{1}{i !}(t-\beta)^{i}\left(\frac{t-\alpha}{\beta-\alpha}\right)^{m} \sum_{k=0}^{n-m-1-i}\left(\begin{array}{c}
m+k-1 \\
k
\end{array}\right)\left(\frac{t-\beta}{\alpha-\beta}\right)^{k}
$$

and also the remainder $R_{(m, n)}(f, t)$ is given by

$$
R_{(m, n)}(f, t)=\int_{\alpha}^{\beta} G_{(m, n)}(t, s) f^{(n)}(s) d s
$$

with

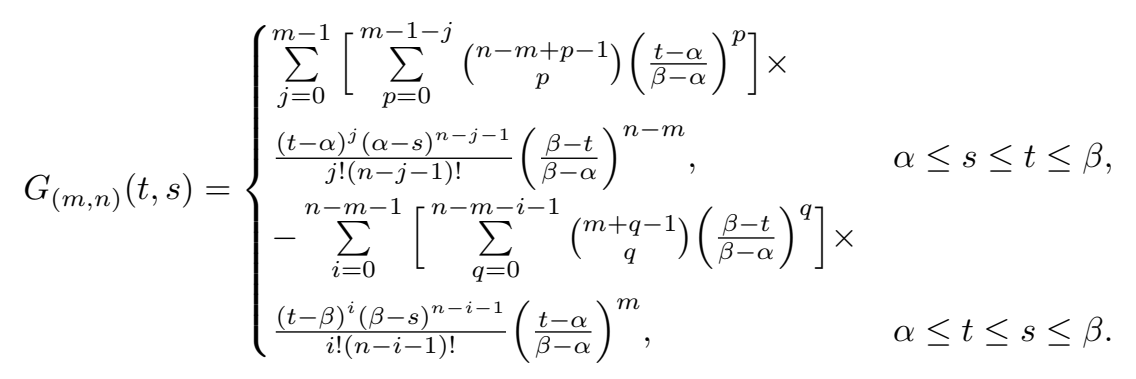

For Type Two-point Taylor conditions, from Theorem 1.4 we have

$$
f(t)=\rho_{2 T}(t)+R_{2 T}(f, t)
$$


where $\rho_{2 T}(t)$ is the two-point Taylor interpolating polynomial i.e,

$$
\begin{array}{r}
\rho_{2 T}(t)=\sum_{i=0}^{m-1} \sum_{k=0}^{m-1-i}\left(\begin{array}{c}
m+k-1 \\
k
\end{array}\right) \\
{\left[\frac{(t-\alpha)^{i}}{i !}\left(\frac{t-\beta}{\alpha-\beta}\right)^{m}\left(\frac{t-\alpha}{\beta-\alpha}\right)^{k} f^{(i)}(\alpha)\right.} \\
\left.+\frac{(t-\beta)^{i}}{i !}\left(\frac{t-\alpha}{\beta-\alpha}\right)^{m}\left(\frac{t-\beta}{\alpha-\beta}\right)^{k} f^{(i)}(\beta)\right]
\end{array}
$$

and the remainder $R_{2 T}(f, t)$ is given by

$$
R_{2 T}(f, t)=\int_{\alpha}^{\beta} G_{2 T}(t, s) f^{(n)}(s) d s
$$

with

$$
G_{2 T}(t, s)= \begin{cases}\frac{(-1)^{m}}{(2 m-1) !} p^{m}(t, s) \sum_{j=0}^{m-1}\left(\begin{array}{c}
m-1+j \\
j
\end{array}\right)(t-s)^{m-1-j} q^{j}(t, s), \quad s \leq t \\
\frac{(-1)^{m}}{(2 m-1) !} q^{m}(t, s) \sum_{j=0}^{m-1}\left(\begin{array}{c}
m-1+j \\
j
\end{array}\right)(s-t)^{m-1-j} p^{j}(t, s), \quad s \geq t\end{cases}
$$

where $p(t, s)=\frac{(s-\alpha)(\beta-t)}{\beta-\alpha}, q(t, s)=p(s, t), \forall t, s \in[\alpha, \beta]$.

The following Lemma describes the positivity of Green's function (1.12) see (Beesack [4] and [Levin [16]).

Lemma 1.1. The Green's function $G_{H, n}(t, s)$ has the following properties:

(i) $\frac{G_{H, n}(t, s)}{w(t)}>0, a_{1} \leq t \leq a_{r}, a_{1} \leq s \leq a_{r}$;

(ii) $G_{H, n}(t, s) \leq \frac{1}{(n-1) !(\beta-\alpha)}|w(t)|$;

(iii) $\int_{\alpha}^{\beta} G_{H, n}(t, s) d s=\frac{w(t)}{n !}$.

We arrange the paper in this manner, in section 2, we use Peano's representation of Hermite interpolating polynomial and newly defined Green functions to establish identities for majorization inequalities. We present generalized majorization inequalities and in particular we discuss the results for $(m, n-m)$ interpolating polynomial and two-point Taylor interpolating polynomial. In section 3, we give bounds for the identities related to the generalizations of majorization inequalities by using $\check{C}$ ebyšev functionals. We also give Grüss type inequalities and Ostrowski-type inequalities for these functionals. In section 4, we present Lagrange and Cauchy type mean value theorems related to the defined functionals and also give $n$-exponential convexity which leads to exponential convexity and then log-convexity. At the end, in section 5, we give some related analytical inequalities to our generalized results of upper bounds and also construct examples of exponentially convex functions. 


\section{Main Results via Peano's representation and new Green functions}

As mentioned in [11], the complete reference about Abel-Gontscharoff polynomial and theorem for 'twopoint right focal' problem is given in [3]:

Remark 2.1. As a special choice the Abel-Gontscharoff polynomial for 'two-point right focal' interpolating polynomial for $n=2$ can be given as:

$$
f(z)=f(\alpha)+(z-\alpha) f^{\prime}(\beta)+\int_{\alpha}^{\beta} G_{\Omega, 2}(z, w) f^{\prime \prime}(w) d w,
$$

where $G_{\Omega, 2}(z, w)$ is the Green's function for 'two-point right focal problem' given as

$$
G_{1}(z, w)=G_{\Omega, 2}(z, w)= \begin{cases}(\alpha-w), & \alpha \leq w \leq z \\ (\alpha-z), & z \leq w \leq \beta\end{cases}
$$

Mehmood et al. (2017) [11] introduced some new types of Green functions by keeping in view AbelGontscharoff Green's function for 'two-point right focal problem' that are:

$$
\begin{aligned}
& G_{2}(z, w)= \begin{cases}(z-\beta), & \alpha \leq w \leq z, \\
(w-\beta), & z \leq w \leq \beta .\end{cases} \\
& G_{3}(z, w)= \begin{cases}(z-\alpha), & \alpha \leq w \leq z, \\
(w-\alpha), & z \leq w \leq \beta .\end{cases} \\
& G_{4}(z, w)= \begin{cases}(\beta-w), & \alpha \leq w \leq z, \\
(\beta-z), & z \leq w \leq \beta .\end{cases}
\end{aligned}
$$

Mehmood et al. (2017) gave the following lemma, using this we obtain the new generalizations of majorization inequality.

Lemma 2.1. Let $f:[\alpha, \beta] \rightarrow \mathbb{R}$ be a twice differentiable function and $G_{c},(c=1,2,3,4)$ be the new Green functions defined above, then along with (2.1) the following identities holds:

$$
\begin{gathered}
f(z)=f(\beta)+(z-\beta) f^{\prime}(\alpha)+\int_{\alpha}^{\beta} G_{2}(z, w) f^{\prime \prime}(w) d w \\
f(z)=f(\beta)-(\beta-\alpha) f^{\prime}(\alpha)+(z-\alpha) f^{\prime}(\alpha)+\int_{\alpha}^{\beta} G_{3}(z, w) f^{\prime \prime}(w) d w \\
f(z)=f(\alpha)-(\beta-\alpha) f^{\prime}(\alpha)-(\beta-z) f^{\prime}(\beta)+\int_{\alpha}^{\beta} G_{4}(z, w) f^{\prime \prime}(w) d w .
\end{gathered}
$$

Equivalent statements between classical weighted majorization inequality and the inequality constructed by newly Green functions are given as: 
Theorem 2.1. Let $\mathbf{x}=\left(x_{1}, \ldots, x_{m}\right), \mathbf{y}=\left(y_{1}, \ldots, y_{m}\right) \in I^{m}$ be two decreasing $m$-tuples and also $\mathbf{w}=$ $\left(w_{1}, \ldots, w_{m}\right)$ be a real $m$-tuple such that satisfying (1.4) and $G_{c}(c=1,2,3,4)$ is defined as in (2.2)-(2.5) respectively. Then the following statements are equivalent:

(i) For every continuous convex function $f:[\alpha, \beta] \rightarrow \mathbb{R}$, then

$$
\sum_{l=1}^{m} w_{l} f\left(y_{l}\right) \leq \sum_{l=1}^{m} w_{l} f\left(x_{l}\right)
$$

(ii) For $s \in[\alpha, \beta]$, the following inequality holds

$$
\sum_{l=1}^{m} w_{l} G_{c}\left(y_{l}, s\right) \leq \sum_{l=1}^{m} w_{l} G_{c}\left(x_{l}, s\right), \quad c=1,2,3,4 .
$$

Moreover, the statements (i) and (ii) are also equivalent if we change the sign of inequality in both inequalities, in (2.9) and (2.10).

Proof. " $(i) \Rightarrow($ ii $)$ " Suppose the statement (i) satisfies. Fix $c=1,2,3,4$, the functions $G_{c}(., s)(s \in[\alpha, \beta])$ are continuous and also convex, implies that these functions hold inequality (2.9) for each fix p, i.e., (2.10) holds.

$"(i i) \Rightarrow(i)$ " Since $f:[\alpha, \beta] \rightarrow \mathbb{R}$ be a convex function, $f \in C^{2}([\alpha, \beta])$ and $(i i)$ holds. Then the representation of the function $f$ in the form $(2.1),(2.6),(2.7)$ and $(2.8)$ for the functions $G_{c}, c=1,2,3,4$ implies that for all $s \in[\alpha, \beta]$,

$$
\begin{aligned}
& \sum_{l=1}^{m} w_{l} f\left(x_{l}\right)-\sum_{l=1}^{m} w_{l} f\left(y_{l}\right) \\
& \quad=\int_{\alpha}^{\beta}\left(\sum_{l=1}^{m} w_{l} G_{c}\left(x_{l}, s\right)-\sum_{l=1}^{m} w_{l} G_{c}\left(y_{l}, s\right)\right) f^{\prime \prime}(s) d s, \quad c=1,2,3,4 .
\end{aligned}
$$

Since $f$ is a convex function, then $f^{\prime \prime}(x) \geq 0$ for all $x \in[\alpha, \beta]$. So, if for every $s \in[\alpha, \beta]$ the inequality (2.10) holds for each $c=1,2,3,4$, then it follows that for every convex function $f:[\alpha, \beta] \rightarrow \mathbb{R}$, with $f \in C^{2}[\alpha, \beta]$, inequality (2.9) holds.

At the end, note that it is not necessary to demand the existence of the second derivative of the function $f$ ( [12], p.172). The differentiability condition can be directly eliminated by using the fact that it is possible to approximate uniformly a continuous convex functions by convex polynomials.

We give some identities related to the generalizations of majorization inequality by using Peano's representation of Hermite's polynomial and new Green functions:

Theorem 2.2. Let $-\infty<\alpha<\beta<\infty$ and $\alpha \leq a_{1}<a_{2} \cdots<a_{r} \leq \beta,(r \geq 2)$ be the given points, and $f \in C^{n}([\alpha, \beta])$ and $\boldsymbol{w}=\left(w_{1}, \ldots, w_{m}\right), \boldsymbol{x}=\left(x_{1}, \ldots, x_{m}\right)$ and $\boldsymbol{y}=\left(y_{1}, \ldots, y_{m}\right)$ be m-tuples such that $x_{l}, y_{l}$ 
$\in[\alpha, \beta], w_{l} \in \mathbb{R}(l=1, \ldots, m)$. Also let $H_{i j}, G_{H, n}$ and $G_{c}(c=1,2,3,4)$ be as defined in (1.10), (1.12) and (2.2)-(2.5) respectively. Then we have the following identities for $c=1,2,3,4$,

$$
\begin{aligned}
& \sum_{l=1}^{m} w_{l} f\left(x_{l}\right)-\sum_{l=1}^{m} w_{l} f\left(y_{l}\right)=\left(\sum_{l=1}^{m} w_{l} x_{l}-\sum_{l=1}^{m} w_{l} y_{l}\right) f^{\prime}(\alpha) \\
& +\int_{\alpha}^{\beta}\left[\sum_{l=1}^{m} w_{l}\left(G_{c}\left(x_{l}, t\right)-G_{c}\left(y_{l}, t\right)\right)\right] \sum_{j=1}^{r} \sum_{i=0}^{k_{j}} f^{(i+2)}\left(a_{j}\right) H_{i j}(t) d t \\
& +\int_{\alpha}^{\beta} f^{(n)}(s)\left[\int_{\alpha}^{\beta}\left[\sum_{l=1}^{m} w_{l}\left(G_{c}\left(x_{l}, t\right)-G_{c}\left(y_{l}, t\right)\right)\right] G_{H, n-2}(t, s) d t\right] d s,
\end{aligned}
$$

where the Peano's kernel (Green's function) is defined as

$$
G_{H, n-2}(t, s)=\left\{\begin{array}{l}
\sum_{j=1}^{l} \sum_{i=0}^{k_{j}} \frac{\left(a_{j}-s\right)^{n-i-3}}{(n-i-3) !} H_{i j}(t) ; s \leq t, \\
-\sum_{j=l+1}^{r} \sum_{i=0}^{k_{j}} \frac{\left(a_{j}-s\right)^{n-i-3}}{(n-i-3) !} H_{i j}(t) ; s \geq t,
\end{array}\right.
$$

for all $a_{l} \leq s \leq a_{l+1} ; l=0, \ldots, r$ with $a_{0}=\alpha$ and $a_{r+1}=\beta$.

Proof. Fix $c=1,2,3,4$, evaluating the identities one by one (2.1), (2.6), (2.7) and (2.8) into majorization difference, we get

$$
\begin{aligned}
& \sum_{l=1}^{m} w_{l} f\left(x_{l}\right)-\sum_{l=1}^{m} w_{l} f\left(y_{l}\right) \\
& \quad=\left(\sum_{l=1}^{m} w_{l} x_{l}-\sum_{l=1}^{m} w_{l} y_{l}\right) f^{\prime}(\alpha)+\int_{\alpha}^{\beta}\left(\sum_{l=1}^{m} w_{l} G_{c}\left(x_{l}, t\right)-\sum_{l=1}^{m} w_{l} G_{c}\left(y_{l}, t\right)\right) f^{\prime \prime}(t) d t .
\end{aligned}
$$

By the Peano's representation of Hermite's interpolatinhg polynomial Theorem 1.4, $f^{\prime \prime}(t)$ can be expressed as

$$
f^{\prime \prime}(t)=\sum_{j=1}^{r} \sum_{i=0}^{k_{j}} H_{i j}(t) f^{(i+2)}\left(a_{j}\right)+\int_{\alpha}^{\beta} G_{H, n-2}(t, s) f^{(n)}(s) d s .
$$

Using (2.16) in (2.15) we get

$$
\begin{aligned}
& \sum_{l=1}^{m} w_{l} \phi\left(x_{l}\right)-\sum_{l=1}^{m} w_{l} \phi\left(y_{l}\right)=\left(\sum_{l=1}^{m} w_{l} x_{l}-\sum_{l=1}^{m} w_{l} y_{l}\right) f^{\prime}(\alpha) \\
& +\int_{\alpha}^{\beta}\left[\sum_{l=1}^{m} w_{l}\left(G_{c}\left(x_{l}, t\right)-G_{c}\left(y_{l}, t\right)\right)\right] \sum_{j=1}^{r} \sum_{i=0}^{k_{j}} f^{(i+2)}\left(a_{j}\right) H_{i j}(t) d t \\
& +\int_{\alpha}^{\beta}\left(\sum_{l=1}^{m} w_{l}\left(G_{c}\left(x_{l}, t\right)-G_{c}\left(y_{l}, t\right)\right)\right)\left(\int_{\alpha}^{\beta} G_{H, n-2}(t, s) f^{(n)}(s) d s\right) d t
\end{aligned}
$$

after applying Fubini's theorm we get (2.13).

Integral version of the above theorem can be stated as: 
Theorem 2.3. Let $-\infty<\alpha<\beta<\infty$ and $\alpha \leq a_{1}<a_{2} \cdots<a_{r} \leq \beta,(r \geq 2)$ be the given points, $f \in C^{n}([\alpha, \beta])$ and $x, y:[a, b] \rightarrow[\alpha, \beta], w:[a, b] \rightarrow \mathbb{R}$ be continuous functions. Also let $H_{i j}, G_{H, n-2}$ and $G_{c}(c=1,2,3,4)$ be as defined in (1.10), (2.14) and (2.2)-(2.5) respectively. Then we have the following identities for $c=1,2,3,4$,

$$
\begin{aligned}
& \int_{a}^{b} w(\tau) f(x(\tau)) d \tau-\int_{a}^{b} w(\tau) f(y(\tau)) d \tau=\left(\int_{a}^{b} w(\tau) x(\tau) d \tau-\int_{a}^{b} w(\tau) y(\tau) d \tau\right) f^{\prime}(\alpha) \\
& +\int_{\alpha}^{\beta}\left[\int_{a}^{b} w(\tau)\left(G_{c}(x(\tau), t)-G_{c}(y(\tau), t)\right) d \tau\right] \sum_{j=1}^{r} \sum_{i=0}^{k_{j}} f^{(i+2)}\left(a_{j}\right) H_{i j}(t) d t \\
& +\int_{\alpha}^{\beta} f^{(n)}(s)\left(\int_{\alpha}^{\beta}\left[\int_{a}^{b} w(\tau)\left(G_{c}(x(\tau), t)-G_{c}(y(\tau), t)\right) d \tau\right] G_{H, n-2}(t, s) d t\right) d s .
\end{aligned}
$$

Theorem 2.4. Let $-\infty<\alpha=a_{1}<a_{2} \cdots<a_{r}=\beta<\infty,(r \geq 2)$ be the given points, $\boldsymbol{w}=\left(w_{1}, \ldots, w_{m}\right)$, $\boldsymbol{x}=\left(x_{1}, \ldots, x_{m}\right)$ and $\boldsymbol{y}=\left(y_{1}, \ldots, y_{m}\right)$ be $m$-tuples such that $x_{l}, y_{l} \in[\alpha, \beta], w_{l} \in \mathbb{R}(l=1, \ldots, m)$ and $H_{i j}$, $G_{c}(c=1,2,3,4)$ be as defined in (1.10) and (2.2)-(2.5) respectively. Let $f:[\alpha, \beta] \rightarrow \mathbb{R}$ be $n$-convex and

$$
\sum_{l=1}^{m} w_{l}\left(G_{c}\left(x_{l}, t\right)-G_{c}\left(y_{l}, t\right)\right) \geq 0, \quad t \in[\alpha, \beta] .
$$

Consider the inequalities for $c=1,2,3,4$,

$$
\begin{aligned}
& \sum_{l=1}^{m} w_{l} f\left(x_{l}\right)-\sum_{l=1}^{m} w_{l} f\left(y_{l}\right) \geq\left(\sum_{l=1}^{m} w_{l} x_{l}-\sum_{l=1}^{m} w_{l} y_{l}\right) f^{\prime}(\alpha) \\
& +\int_{\alpha}^{\beta}\left[\sum_{l=1}^{m} w_{l}\left(G_{c}\left(x_{l}, t\right)-G_{c}\left(y_{l}, t\right)\right)\right] \sum_{j=1}^{r} \sum_{i=0}^{k_{j}} f^{(i+2)}\left(a_{j}\right) H_{i j}(t) d t .
\end{aligned}
$$

(i) If $k_{j}$ is odd for each $j=2, . ., r$, then the inequalities for $c=1,2,3,4$, in (2.19) hold.

(ii) If $k_{j}$ is odd for each $j=2, . ., r-1$ and $k_{r}$ is even, then the reverse inequalities for $c=1,2,3,4$, in (2.19) hold.

Proof. (i) Since the function $f$ is $n$-convex, therefore without loss of generality we can assume that $\phi$ is $n$-times differentiable and $f^{(n)} \geq 0$ see [13, p. 16 and p. 293]. Also the given condition is that $k_{j}$ is odd for each $j=1,2, . ., r$ implies that

$$
\omega(t)=\prod_{j=1}^{r}\left(t-a_{j}\right)^{k_{j}+1} \geq 0 .
$$

By using the first part of Lemma 1.1 we have that the Peano's kernel $G_{H, n-2}(t, s) \geq 0$. Hence, we can apply Theorem 2.2 to obtain (2.19).

(ii) If $k_{r}$ is even then $\left(t-a_{r}\right)^{k_{r}+1} \leq 0$ for any $t \in[\alpha, \beta]$. Also clearly $\left(t-a_{1}\right)^{k_{1}+1} \geq 0$ for any $t \in[\alpha, \beta]$ and $\prod_{j=2}^{r-1}\left(t-a_{j}\right)^{k_{j}+1} \geq 0$ for $t \in[\alpha, \beta]$ if $k_{j}$ is odd for each $j=2, . ., r-1$, therefore combining all these we have $\omega(t)=\prod_{j=1}^{r}\left(t-a_{j}\right)^{k_{j}+1} \leq 0$ for any $t \in[\alpha, \beta]$ and by using the first part of Lemma 1.1 we have $G_{H, n-2}(t, s) \leq 0$. Hence, we can apply Theorem 2.2 to obtain reverse inequality in (2.19). 
Integral version of the above theorem can be stated as:

Theorem 2.5. Let $-\infty<\alpha=a_{1}<a_{2} \cdots<a_{r}=\beta<\infty,(r \geq 2)$ be given points and $x, y:[a, b] \rightarrow[\alpha, \beta]$, $w:[a, b] \rightarrow \mathbb{R}$ be continuous functions and $H_{i j}$ and $G_{c}(c=1,2,3,4)$ be as defined in (1.10) and (2.2)-(2.5) respectively. Let $f:[\alpha, \beta] \rightarrow \mathbb{R}$ be $n$-convex and

$$
\int_{a}^{b} w(\tau)\left(G_{c}(x(\tau), t)-G_{c}(y(\tau), t)\right) d \tau \geq 0, \quad t \in[\alpha, \beta] .
$$

Consider the inequalities for $c=1,2,3,4$,

$$
\begin{aligned}
& \int_{a}^{b} w(\tau) f(x(\tau)) d \tau-\int_{a}^{b} w(\tau) f(y(\tau)) d \tau \geq\left(\int_{a}^{b} w(\tau) x(\tau) d \tau-\int_{a}^{b} w(\tau) y(\tau) d \tau\right) f^{\prime}(\alpha) \\
& +\int_{\alpha}^{\beta}\left[\int_{a}^{b} w(\tau)\left(G_{c}(x(\tau), t)-G_{c}(y(\tau), t)\right) d \tau\right] \sum_{j=1}^{r} \sum_{i=0}^{k_{j}} f^{(i+2)}\left(a_{j}\right) H_{i j}(t) d t
\end{aligned}
$$

(i) If $k_{j}$ is odd for each $j=2, . ., r$, then the inequalities for $c=1,2,3,4$, in (2.21) hold.

(ii) If $k_{j}$ is odd for each $j=2, . ., r-1$ and $k_{r}$ is even, then the reverse inequalities for $c=1,2,3,4$, in (2.21) hold.

By using type $(m, n-m)$ conditions we can give the following result.

Corollary 2.1. Let $[\alpha, \beta]$ be an interval and $\boldsymbol{w}=\left(w_{1}, \ldots, w_{p}\right), \boldsymbol{x}=\left(x_{1}, \ldots, x_{p}\right)$ and $\boldsymbol{y}=\left(y_{1}, \ldots, y_{p}\right)$ be $p$-tuples such that $x_{l}, y_{l} \in[\alpha, \beta], w_{l} \in \mathbb{R}(l=1, \ldots, p)$. Let $G_{c}(c=1,2,3,4)$ be the Green functions as defined in (2.2)-(2.5) respectively and also $\tau_{i}, \eta_{i}$ be as defined in (1.14) and (1.15) respectively. Let $f:[\alpha, \beta] \rightarrow \mathbb{R}$ be $n$-convex and the inequality (2.18) holds for $p$-tuples. Consider the inequalities for $c=1,2,3,4$,

$$
\begin{aligned}
& \sum_{l=1}^{p} w_{l} \phi\left(x_{l}\right)-\sum_{l=1}^{p} w_{l} \phi\left(y_{l}\right) \geq\left(\sum_{l=1}^{p} w_{l} x_{l}-\sum_{l=1}^{p} w_{l} y_{l}\right) f^{\prime}(\alpha) \\
& \quad+\int_{\alpha}^{\beta}\left[\sum_{l=1}^{p} w_{l}\left(G_{c}\left(x_{l}, t\right)-G_{c}\left(y_{l}, t\right)\right)\right]\left(\sum_{i=0}^{m-1} \tau_{i}(t) f^{(i+2)}(\alpha)+\sum_{i=0}^{n-m-1} \eta_{i}(t) f^{(i+2)}(\beta)\right) d t .
\end{aligned}
$$

(i) If $n-m$ is even, then the inequalities for $c=1,2,3$, 4, in (2.22) hold.

(ii) If $n-m$ is odd, then the reverse inequalities for $c=1,2,3,4$, in (2.22) hold.

By using Two-point Taylor conditions we can give the following result.

Corollary 2.2. Let $[\alpha, \beta]$ be an interval, $\boldsymbol{w}=\left(w_{1}, \ldots, w_{p}\right), \boldsymbol{x}=\left(x_{1}, \ldots, x_{p}\right)$ and $\boldsymbol{y}=\left(y_{1}, \ldots, y_{p}\right)$ be $p$-tuples such that $x_{l}, y_{l} \in[\alpha, \beta], w_{l} \in \mathbb{R}(l=1, \ldots, p)$ and $G_{c}(c=1,2,3,4)$ be the green function as defined in (2.2)(2.5) respectively. Let $f:[\alpha, \beta] \rightarrow \mathbb{R}$ be $n$-convex and the inequality (2.18) holds for $p$-tuples. Consider the 
inequalities for $c=1,2,3,4$,

$$
\begin{aligned}
& \sum_{l=1}^{p} w_{l} f\left(x_{l}\right)-\sum_{l=1}^{p} w_{l} f\left(y_{l}\right) \geq\left(\sum_{l=1}^{p} w_{l} x_{l}-\sum_{l=1}^{p} w_{l} y_{l}\right) f^{\prime}(\alpha) \\
& +\int_{\alpha}^{\beta}\left[\sum_{l=1}^{p} w_{l}\left(G_{c}\left(x_{l}, t\right)-G_{c}\left(y_{l}, t\right)\right)\right]\left[\sum _ { i = 0 } ^ { m - 1 } \sum _ { k = 0 } ^ { m - 1 - i } ( \begin{array} { c } 
{ m + k - 1 } \\
{ k }
\end{array} ) \left[\frac{(t-\alpha)^{i}}{i !}\left(\frac{t-\beta}{\alpha-\beta}\right)^{m}\left(\frac{t-\alpha}{\beta-\alpha}\right)^{k} f^{(i+2)}(\alpha)\right.\right. \\
& \left.\left.\quad+\frac{(t-\beta)^{i}}{i !}\left(\frac{t-\alpha}{\beta-\alpha}\right)^{m}\left(\frac{t-\beta}{\alpha-\beta}\right)^{k} f^{(i+2)}(\beta)\right]\right] d t . \quad(2.23)
\end{aligned}
$$

(i) If $m$ is even, then the inequalities for $c=1,2,3,4$, in (2.23) hold.

(ii) If $m$ is odd, then the reverse inequalities for $c=1,2,3,4$, in (2.23) hold.

Remark 2.2. Similarly we can give integral version of Corollaries 2.1,2.2.

The following generalization of classical majorization theorem (also known as Karamata's inequality) is valid.

Theorem 2.6. Let $-\infty<\alpha=a_{1}<a_{2} \cdots<a_{r}=\beta<\infty,(r \geq 2)$ be the given points, $\boldsymbol{x}=\left(x_{1}, \ldots, x_{m}\right)$ and $\boldsymbol{y}=\left(y_{1}, \ldots, y_{m}\right)$ be $m$-tuples such that $\mathbf{y} \prec \mathbf{x}$ with $x_{l}, y_{l} \in[\alpha, \beta](l=1, \ldots, m)$. Let $H_{i j}$ and $G_{c}(c=1,2,3,4)$ be as defined in (1.10) and (2.2)-(2.5) respectively and also $f:[\alpha, \beta] \rightarrow \mathbb{R}$ be $n$-convex. Consider the inequalities for $c=1,2,3,4$,

$$
\sum_{l=1}^{m} f\left(x_{l}\right)-\sum_{l=1}^{m} f\left(y_{l}\right) \geq \int_{\alpha}^{\beta}\left[\sum_{l=1}^{m}\left(G_{c}\left(x_{l}, t\right)-G_{c}\left(y_{l}, t\right)\right)\right] \sum_{j=1}^{r} \sum_{i=0}^{k_{j}} f^{(i+2)}\left(a_{j}\right) H_{i j}(t) d t .
$$

(i) If $k_{j}$ is odd for each $j=2, . ., r$, then the inequalities for $c=1,2,3,4$, in (2.24) hold.

(ii) If $k_{j}$ is odd for each $j=2, . ., r-1$ and $k_{r}$ is even, then the reverse inequalities for $c=1,2,3,4$, in (2.24) hold.

If the inequalities (reverse inequalities) for $c=1,2,3,4$, in (2.24) hold and the function $\mathbb{F}()=.\sum_{j=1}^{r} \sum_{i=0}^{k_{j}} f^{(i+2)}\left(a_{j}\right) H_{i j}($.$) is non negative ( non positive), then the right hand side of (2.24) will be non$ negative (non positive) for each $c=1,2,3,4$, that is the inequality (reverse inequality) in (1.2) will hold.

Proof. (i) Since the function $G$ is convex and $\mathbf{y} \prec \mathbf{x}$ therefore by Theorem 1.1, the inequalities for $c=1,2,3,4$, in (2.18) hold for $w_{l}=1$. Hence by Theorem 2.4(i) the inequalities for $c=1,2,3,4$, in (2.24) hold. Also if the function $\mathbb{F}$ is convex then by using $\mathbb{F}$ in (1.2) instead of $f$ we get that the right hand side of (2.24) is non negative for each $c=1,2,3,4$.

Similarly we can prove part (ii).

In the following theorem we give generalization of Fuch's majorization theorem. 
Theorem 2.7. Let $-\infty<\alpha=a_{1}<a_{2} \cdots<a_{r}=\beta<\infty,(r \geq 2)$ be the given points, $\boldsymbol{x}=\left(x_{1}, \ldots, x_{m}\right)$ and $\boldsymbol{y}=\left(y_{1}, \ldots, y_{m}\right)$ be decreasing $m$-tuples and $\boldsymbol{w}=\left(w_{1}, \ldots, w_{m}\right)$ be any $m$-tuple with $x_{l}, y_{l} \in[\alpha, \beta], w_{l} \in \mathbb{R}$ $(l=1, \ldots, m)$ which satisfy (1.3) and (1.4). Let $H_{i j}$ and $G_{c}(c=1,2,3,4)$ be as defined in (1.10) and (2.2)-(2.5) respectively and also $f:[\alpha, \beta] \rightarrow \mathbb{R}$ be $n$-convex, then

$$
\sum_{l=1}^{m} w_{l} f\left(x_{l}\right)-\sum_{l=1}^{m} w_{l} f\left(y_{l}\right) \geq \int_{\alpha}^{\beta}\left[\sum_{l=1}^{m} w_{l}\left(G_{c}\left(x_{l}, t\right)-G_{c}\left(y_{l}, t\right)\right)\right] \sum_{j=1}^{r} \sum_{i=0}^{k_{j}} f^{(i+2)}\left(a_{j}\right) H_{i j}(t) d t .
$$

(i) If $k_{j}$ is odd for each $j=2, . ., r$, then the inequalities for $c=1,2,3,4$, in (2.25) hold.

(ii) If $k_{j}$ is odd for each $j=2, . ., r-1$ and $k_{r}$ is even, then the reverse inequalities for $c=1,2,3,4$, in (2.25) hold.

If the inequalities (reverse inequalities) for $c=1,2,3,4$, in (2.25) hold and the function $\mathbb{F}()=.\sum_{j=1}^{r} \sum_{i=0}^{k_{j}} f^{(i+2)}\left(a_{j}\right) H_{i j}($.$) is non negative (non positive), then the right hand side of (2.25) will be non$ negative (non positive) for $c=1,2,3,4$, that is the inequality (reverse inequality) in (1.5) will hold.

Proof. Similar to the proof of Theorem 2.6.

In the following theorem we give generalized majorization integral inequality.

Theorem 2.8. Let $-\infty<\alpha=a_{1}<a_{2} \cdots<a_{r}=\beta<\infty,(r \geq 2)$ be the given points, and $x, y:[a, b] \rightarrow$ $[\alpha, \beta]$ be decreasing and $w:[a, b] \rightarrow \mathbb{R}$ be continuous functions such that (1.6) and (1.7) hold. Also let $H_{i j}$ and $G_{c}(c=1,2,3,4)$ be as defined in (1.10) and (2.2)-(2.5) respectively and also $f:[\alpha, \beta] \rightarrow \mathbb{R}$ be $n$-convex and consider the inequalities for $c=1,2,3,4$,

$$
\begin{aligned}
& \int_{a}^{b} w(\tau) f(x(\tau)) d \tau-\int_{a}^{b} w(\tau) f(y(\tau)) d \tau \\
& \geq \int_{\alpha}^{\beta}\left[\int_{a}^{b} w(\tau)\left(G_{c}(x(\tau), t)-G_{c}(y(\tau), t)\right) d \tau\right] \sum_{j=1}^{r} \sum_{i=0}^{k_{j}} f^{(i+2)}\left(a_{j}\right) H_{i j}(t) d t
\end{aligned}
$$

(i) If $k_{j}$ is odd for each $j=2, . ., r$, then the inequalities for $c=1,2,3,4$, in (2.26) hold.

(ii) If $k_{j}$ is odd for each $j=2, . ., r-1$ and $k_{r}$ is even, then the reverse inequalities for $c=1,2,3,4$, in (2.26) hold.

If the inequalities (reverse inequalities) for $c=1,2,3,4$, in (2.26) hold and the function $\mathbb{F}()=.\sum_{j=1}^{r} \sum_{i=0}^{k_{j}} f^{(i+2)}\left(a_{j}\right) H_{i j}($.$) is non negative (non positive), then the right hand side of (2.26) will be non$ negative (non positive) for $c=1,2,3,4$, that is the inequality (reverse inequality) in (1.8) will hold.

By using type $(m, n-m)$ conditions we can give generalization of majorization inequality for majorized tuples: 
Corollary 2.3. Let $[\alpha, \beta]$ be an interval, $\boldsymbol{x}=\left(x_{1}, \ldots, x_{p}\right)$ and $\boldsymbol{y}=\left(y_{1}, \ldots, y_{p}\right)$ be any $p$-tuple such that $\mathbf{y}$ $\prec \mathbf{x}$ with $x_{l}, y_{l} \in[\alpha, \beta](l=1, \ldots, p)$. Let $\tau_{i}$ and $\eta_{i}$ be as defined in (1.14) and (1.15) respectively. Let $G_{c}(c=1,2,3,4)$ be defined as in (2.2)-(2.5) respectively and also $f:[\alpha, \beta] \rightarrow \mathbb{R}$ be $n$-convex. Consider the inequalities for $c=1,2,3,4$,

$$
\begin{aligned}
& \sum_{l=1}^{p} f\left(x_{l}\right)-\sum_{l=1}^{p} f\left(y_{l}\right) \\
& \quad \geq \int_{\alpha}^{\beta}\left[\sum_{l=1}^{p}\left(G_{c}\left(x_{l}, t\right)-G_{c}\left(y_{l}, t\right)\right)\right]\left(\sum_{i=0}^{m-1} \tau_{i}(t) f^{(i+2)}(\alpha)+\sum_{i=0}^{n-m-1} \eta_{i}(t) f^{(i+2)}(\beta)\right) d t .
\end{aligned}
$$

(i) If $n-m$ is even, then the inequalities for $c=1,2,3,4$ in (2.27) hold.

(ii) If $n-m$ is odd, then the reverse inequalities for $c=1,2,3,4$, in (2.27) hold.

If the inequalities (reverse inequalities) for $c=1,2,3,4$, in (2.27) hold and the function

$\mathbb{F}()=.\sum_{i=0}^{m-1} f^{(i+2)}(\alpha) \tau_{i}()+.\sum_{i=0}^{n-m-1} f^{(i+2)}(\beta) \eta_{i}($.$) is non negative (non positive), then the right hand side$ of (2.27) will be non negative (non positive) for each $c=1,2,3,4$, that is the inequality (reverse inequality) in (1.2) will hold.

By using Two-point Taylor conditions we can give generalization of majorization inequality for majorized tuples:

Corollary 2.4. Let $[\alpha, \beta]$ be an interval and $\boldsymbol{x}=\left(x_{1}, \ldots, x_{p}\right), \boldsymbol{y}=\left(y_{1}, \ldots, y_{p}\right)$ be decreasing $p$-tuples such that $\mathbf{y} \prec \mathbf{x}$ with $x_{l}, y_{l} \in[\alpha, \beta](l=1, \ldots, p)$. Let $f:[\alpha, \beta] \rightarrow \mathbb{R}$ be $n$-convex. Consider the inequalities for $c=1,2,3,4$,

$$
\begin{aligned}
\sum_{l=1}^{p} f\left(x_{l}\right)-\sum_{l=1}^{p} f\left(y_{l}\right) \geq \int_{\alpha}^{\beta} & {\left[\sum_{l=1}^{p}\left(G_{c}\left(x_{l}, t\right)-G_{c}\left(y_{l}, t\right)\right)\right] \mathbb{F}(t) d t, } \\
\text { where } \mathbb{F}(t)=\sum_{i=0}^{m-1} \sum_{k=0}^{m-1-i}\left(\begin{array}{c}
m+k-1 \\
k
\end{array}\right) & {\left[\frac{(t-\alpha)^{i}}{i !}\left(\frac{t-\beta}{\alpha-\beta}\right)^{m}\left(\frac{t-\alpha}{\beta-\alpha}\right)^{k} f^{(i+2)}(\alpha)\right.} \\
+ & \left.\frac{(t-\beta)^{i}}{i !}\left(\frac{t-\alpha}{\beta-\alpha}\right)^{m}\left(\frac{t-\beta}{\alpha-\beta}\right)^{k} f^{(i+2)}(\beta)\right] .
\end{aligned}
$$

(i) If $m$ is even, then the inequalities for $c=1,2,3,4$, in (2.28) hold.

(ii) If $m$ is odd, then the reverse inequalities for $c=1,2,3,4$, in (2.28) hold.

If the inequalities (reverse inequalities) for $c=1,2,3,4$, in (2.28) hold and the function $\mathbb{F}($.$) is non negative$ (non positive), then the right hand side of (2.28) will be non negative (non positive) for each $c=1,2,3,4$, that is the inequality (reverse inequality) in (1.2) will hold.

By using type $(m, n-m)$ conditions we can give the following weighted majorization inequality. 
Corollary 2.5. Let $[\alpha, \beta]$ be an interval and $\boldsymbol{x}=\left(x_{1}, \ldots, x_{p}\right)$ and $\boldsymbol{y}=\left(y_{1}, \ldots, y_{p}\right)$ be decreasing $p$-tuples and $\boldsymbol{w}=\left(w_{1}, \ldots, w_{p}\right)$ be any $p$-tuple such that $x_{l}, y_{l} \in[\alpha, \beta], w_{l} \in \mathbb{R}(l=1, \ldots, p)$ which satisfy (1.3) and (1.4). Let $\tau_{i}$ and $\eta_{i}$ be as defined in (1.14) and (1.15) respectively and let $f:[\alpha, \beta] \rightarrow \mathbb{R}$ be $n$-convex. Consider the inequalities for $c=1,2,3,4$,

$$
\begin{aligned}
& \sum_{l=1}^{p} w_{l} f\left(x_{l}\right)-\sum_{l=1}^{p} w_{l} f\left(y_{l}\right) \\
& \quad \geq \int_{\alpha}^{\beta}\left[\sum_{l=1}^{p} w_{l}\left(G_{c}\left(x_{l}, t\right)-G_{c}\left(y_{l}, t\right)\right)\right]\left(\sum_{i=0}^{m-1} \tau_{i}(t) f^{(i+2)}(\alpha)+\sum_{i=0}^{n-m-1} \eta_{i}(t) f^{(i+2)}(\beta)\right) d t .
\end{aligned}
$$

(i) If $n-m$ is even, then the inequalities for $c=1,2,3,4$, in (2.29) hold.

(ii) If $n-m$ is odd, then the reverse inequalities for $c=1,2,3,4$, in (2.29) hold.

If the inequalities (reverse inequalities) for $c=1,2,3,4$, in (2.29) hold and the function

$\mathbb{F}()=.\sum_{i=0}^{m-1} f^{(i+2)}(\alpha) \tau_{i}()+.\sum_{i=0}^{n-m-1} f^{(i+2)}(\beta) \eta_{i}($.$) is non negative (non positive), then the right hand side$ of (2.29) will be non negative (non positive) for each $c=1,2,3,4$, that is the inequality (reverse inequality) in (1.5) will hold.

By using Two-point Taylor conditions we can give the following weighted majorization inequality.

Corollary 2.6. Let $[\alpha, \beta]$ be an interval and $\boldsymbol{x}=\left(x_{1}, \ldots, x_{p}\right), \boldsymbol{y}=\left(y_{1}, \ldots, y_{p}\right)$ be decreasing $p$-tuples such that $x_{l}, y_{l} \in[\alpha, \beta], w_{l} \in \mathbb{R}(l=1, \ldots, p)$ which satisfy (1.3) and (1.4) and let $f:[\alpha, \beta] \rightarrow \mathbb{R}$ be $n$-convex. Consider the inequalities for $c=1,2,3,4$,

$$
\begin{aligned}
\sum_{l=1}^{p} w_{l} f\left(x_{l}\right)-\sum_{l=1}^{p} w_{l} f\left(y_{l}\right) \geq \int_{\alpha}^{\beta} & {\left[\sum_{l=1}^{p} w_{l}\left(G_{c}\left(x_{l}, t\right)-G_{c}\left(y_{l}, t\right)\right)\right] \mathbb{F}(t) d t, } \\
\text { where } \mathbb{F}(t)=\sum_{i=0}^{m-1} \sum_{k=0}^{m-1-i}\left(\begin{array}{c}
m+k-1 \\
k
\end{array}\right) & {\left[\frac{(t-\alpha)^{i}}{i !}\left(\frac{t-\beta}{\alpha-\beta}\right)^{m}\left(\frac{t-\alpha}{\beta-\alpha}\right)^{k} f^{(i+2)}(\alpha)\right.} \\
+ & \left.\frac{(t-\beta)^{i}}{i !}\left(\frac{t-\alpha}{\beta-\alpha}\right)^{m}\left(\frac{t-\beta}{\alpha-\beta}\right)^{k} f^{(i+2)}(\beta)\right] .
\end{aligned}
$$

(i) If $m$ is even, then the inequalities for $c=1,2,3,4$, in (2.30) hold.

(ii) If $m$ is odd, then the reverse inequalities for $c=1,2,3,4$, in (2.30) hold.

If the inequalities (reverse inequalities) for $c=1,2,3,4$, in (2.30) hold and the function $\mathbb{F}($.$) is non negative$ (non positive), then the right hand side of (2.30) will be non negative (non positive) for each $c=1,2,3,4$, that is the inequality (reverse inequality) in (1.5) will hold.

The integral version of the above Corollaries can be stated as: 
Corollary 2.7. Let $[\alpha, \beta]$ be an interval and $x, y:[a, b] \rightarrow[\alpha, \beta]$ be decreasing and $w:[a, b] \rightarrow \mathbb{R}$ be continuous function such that (1.6), (1.7) hold. Let $\tau_{i}$ and $\eta_{i}$ be as defined in (1.14) and (1.15) respectively and $f:[\alpha, \beta] \rightarrow \mathbb{R}$ be $n$-convex. Consider the inequalities for $c=1,2,3,4$,

$$
\begin{aligned}
& \int_{a}^{b} w(\tau) f(x(\tau)) d \tau-\int_{a}^{b} w(\tau) f(y(\tau)) d \tau \\
& \quad \geq \int_{\alpha}^{\beta}\left[\int_{a}^{b} w(\tau)\left(G_{c}(x(\tau), t)-G_{c}(y(\tau), t)\right) d \tau\right]\left(\sum_{i=0}^{m-1} \tau_{i}(t) f^{(i+2)}(\alpha)+\sum_{i=0}^{n-m-1} \eta_{i}(t) f^{(i+2)}(\beta)\right) d t
\end{aligned}
$$

(i) If $n-m$ is even, then the inequalities for $c=1,2,3,4$, in (2.31) hold.

(ii) If $n-m$ is odd, then the reverse inequalities for $c=1,2,3,4$, in (2.31) hold.

If the inequalities (reverse inequalities) for $c=1,2,3,4$, in (2.31) holds and the function $\mathbb{F}()=.\sum_{i=0}^{m-1} f^{(i+2)}(\alpha) \tau_{i}()+.\sum_{i=0}^{n-m-1} f^{(i+2)}(\beta) \eta_{i}($.$) is non negative (non positive), then the right hand side$ of (2.31) will be non negative (non positive) for each $c=1,2,3,4$, that is the inequality (reverse inequality) in (1.8) will hold.

Corollary 2.8. Let $[\alpha, \beta]$ be an interval and $x, y:[a, b] \rightarrow[\alpha, \beta]$ be decreasing and $w:[a, b] \rightarrow \mathbb{R}$ be continuous functions such that (1.6) and (1.7) hold. Let $f:[\alpha, \beta] \rightarrow \mathbb{R}$ be $n$-convex. Consider the inequalities for $c=1,2,3,4$,

$$
\begin{aligned}
\int_{a}^{b} w(\tau) f(x(\tau)) d \tau-\int_{a}^{b} w(\tau) f(y(\tau)) d \tau \geq \int_{\alpha}^{\beta} & {\left[\int_{a}^{b} w(\tau)\left(G_{c}(x(\tau), t)-G_{c}(y(\tau), t)\right) d \tau\right] F(t) d t } \\
\text { where } \mathbb{F}(t)= & \sum_{i=0}^{m-1} \sum_{k=0}^{m-1-i}\left(\begin{array}{c}
m+k-1 \\
k
\end{array}\right)\left[\frac{(t-\alpha)^{i}}{i !}\left(\frac{t-\beta}{\alpha-\beta}\right)^{m}\left(\frac{t-\alpha}{\beta-\alpha}\right)^{k} f^{(i+2)}(\alpha)\right. \\
& \left.+\frac{(t-\beta)^{i}}{i !}\left(\frac{t-\alpha}{\beta-\alpha}\right)^{m}\left(\frac{t-\beta}{\alpha-\beta}\right)^{k} f^{(i+2)}(\beta)\right]
\end{aligned}
$$

(i) If $m$ is even, then the inequalities for $c=1,2,3,4$, in (2.32) hold.

(ii) If $m$ is odd, then the reverse inequalities for $c=1,2,3,4$, in (2.32) hold.

If the inequalities (reverse inequalities) for $c=1,2,3,4$, in (2.32) hold and the function $\mathbb{F}($.$) is non negative$ (non positive), then the right hand side of (2.32) will be non negative (non positive) for each $c=1,2,3,4$, that is the inequality (reverse inequality) in (1.8) will hold.

\section{UPPER BOUNDS FOR OBTAINED GENERALIZED IDENTITIES}

For two Lebesgue integrable functions $f, h:[\alpha, \beta] \rightarrow \mathbb{R}$ we consider the Čebyšev functional

$$
\Lambda(f, h)=\frac{1}{\beta-\alpha} \int_{\alpha}^{\beta} f(t) h(t) d t-\frac{1}{\beta-\alpha} \int_{\alpha}^{\beta} f(t) d t \cdot \frac{1}{\beta-\alpha} \int_{\alpha}^{\beta} h(t) d t .
$$

In [6] the authors proved the following theorems: 
Theorem 3.1. Let $f:[\alpha, \beta] \rightarrow \mathbb{R}$ be a Lebesgue integrable function and $h:[\alpha, \beta] \rightarrow \mathbb{R}$ be an absolutely continuous function with $(\cdot-\alpha)(\beta-\cdot)\left[h^{\prime}\right]^{2} \in L[\alpha, \beta]$. Then we have the inequality

$$
|\Lambda(f, h)| \leq \frac{1}{\sqrt{2}}[\Lambda(f, f)]^{\frac{1}{2}} \frac{1}{\sqrt{\beta-\alpha}}\left(\int_{\alpha}^{\beta}(x-\alpha)(\beta-x)\left[h^{\prime}(x)\right]^{2} d x\right)^{\frac{1}{2}} .
$$

The constant $\frac{1}{\sqrt{2}}$ in (3.1) is the best possible.

Theorem 3.2. Assume that $h:[\alpha, \beta] \rightarrow \mathbb{R}$ is monotonic nondecreasing on $[\alpha, \beta]$ and $f:[\alpha, \beta] \rightarrow \mathbb{R}$ is absolutely continuous with $f^{\prime} \in L_{\infty}[\alpha, \beta]$. Then we have the inequality

$$
|\Lambda(f, h)| \leq \frac{1}{2(\beta-\alpha)}\left\|f^{\prime}\right\|_{\infty} \int_{\alpha}^{\beta}(x-\alpha)(\beta-x) d h(x) .
$$

The constant $\frac{1}{2}$ in (3.2) is the best possible.

In this section, we give the upper bounds like Grüss-type and Ostrowski-type for our generalized results.

For $m$-tuples $\mathbf{w}=\left(w_{1}, \ldots, w_{m}\right), \mathbf{x}=\left(x_{1}, \ldots, x_{m}\right)$ and $\mathbf{y}=\left(y_{1}, \ldots, y_{m}\right)$ with $x_{l}, y_{l} \in[\alpha, \beta], w_{l} \in \mathbb{R}(l=$ $1, \ldots, m)$ and the Green functions $G_{c}(c=1,2,3,4)$ and $G_{H, n-2}$ be as defined in (2.2)-(2.5) and (2.14) respectively, denote

$$
\mathfrak{L}(s)=\int_{\alpha}^{\beta}\left[\sum_{l=1}^{m} w_{l}\left(G_{c}\left(x_{l}, t\right)-G_{c}\left(y_{l}, t\right)\right)\right] G_{H, n-2}(t, s) d t, \quad s \in[\alpha, \beta],
$$

for $c=1,2,3,4$, similarly for continuous functions $x, y:[a, b] \rightarrow[\alpha, \beta], w:[a, b] \rightarrow \mathbb{R}$ and the Green function $G_{c}(c=1,2,3,4)$ and $G_{H, n-2}$ be as defined in (2.2)-(2.5) and (2.14) respectively, denote

$$
\mathfrak{J}(s)=\int_{\alpha}^{\beta}\left[\int_{a}^{b} w(\tau)\left(G_{c}(x(\tau), t)-G_{c}(y(\tau), t)\right) d \tau\right] G_{H, n-2}(t, s) d t, \quad s \in[\alpha, \beta],
$$

for $c=1,2,3,4$.

Consider the Čebyšev functionals $\Lambda(\mathfrak{L}, \mathfrak{L}), \Lambda(\mathfrak{J}, \mathfrak{J})$ are given by:

$$
\begin{gathered}
\Lambda(\mathfrak{L}, \mathfrak{L})=\frac{1}{\beta-\alpha} \int_{\alpha}^{\beta} \mathfrak{L}^{2}(s) d s-\left(\frac{1}{\beta-\alpha} \int_{\alpha}^{\beta} \mathfrak{L}(s) d s\right)^{2}, \\
\Lambda(\mathfrak{J}, \mathfrak{J})=\frac{1}{\beta-\alpha} \int_{\alpha}^{\beta} \mathfrak{J}^{2}(s) d s-\left(\frac{1}{\beta-\alpha} \int_{\alpha}^{\beta} \mathfrak{J}(s) d s\right)^{2} .
\end{gathered}
$$

Theorem 3.3. Let $-\infty<\alpha<\beta<\infty$ and $\alpha \leq a_{1}<a_{2} \cdots<a_{r} \leq \beta,(r \geq 2)$ be the given points, and $f \in C^{n}([\alpha, \beta])$ such that $(\cdot-\alpha)(\beta-\cdot)\left[f^{(n+1)}\right]^{2} \in L[\alpha, \beta]$ and $\boldsymbol{w}=\left(w_{1}, \ldots, w_{m}\right), \boldsymbol{x}=\left(x_{1}, \ldots, x_{m}\right)$ and $\boldsymbol{y}=\left(y_{1}, \ldots, y_{m}\right)$ be $m$-tuples such that $x_{l}, y_{l} \in[\alpha, \beta], w_{l} \in \mathbb{R}(l=1, \ldots, m)$. Also let $H_{i j}$ be the fundamental 
polynomials of the Hermite basis and the functions $G_{c}(c=1,2,3,4)$ and $\mathfrak{L}$ be defined by (2.2)-(2.5) and (3.3) respectively. Then

$$
\begin{array}{r}
\sum_{l=1}^{m} w_{l} f\left(x_{l}\right)-\sum_{l=1}^{m} w_{l} f\left(y_{l}\right)=\left(\sum_{l=1}^{m} w_{l} x_{l}-\sum_{l=1}^{m} w_{l} y_{l}\right) f^{\prime}(\alpha) \\
+\sum_{j=1}^{r} \sum_{i=0}^{k_{j}} \phi^{(i+2)}\left(a_{j}\right) \int_{\alpha}^{\beta}\left[\sum_{l=1}^{m} w_{l}\left(G_{c}\left(x_{l}, t\right)-G_{c}\left(y_{l}, t\right)\right)\right] H_{i j}(t) d t \\
+\frac{f^{(n-1)}(\beta)-f^{(n-1)}(\alpha)}{\beta-\alpha} \int_{\alpha}^{\beta} \mathfrak{L}(s) d s+\mathbb{R} \mathbb{E M}(f ; \alpha, \beta),
\end{array}
$$

where the remainder $\mathbb{R} \mathbb{E M}(f ; \alpha, \beta)$ satisfies the estimation

$$
|\mathbb{R E M}(f ; \alpha, \beta)| \leq \frac{\sqrt{\beta-\alpha}}{\sqrt{2}}[\Lambda(\mathfrak{L}, \mathfrak{L})]^{\frac{1}{2}}\left|\int_{\alpha}^{\beta}(s-\alpha)(\beta-s)\left[f^{(n+1)}(s)\right]^{2} d s\right|^{\frac{1}{2}} .
$$

Proof. Comparing (3.7) and (2.13) we have

$$
\mathbb{R} \mathbb{E M}(f ; \alpha, \beta)=(\beta-\alpha) \Lambda\left(\mathfrak{L}, f^{(n)}\right) .
$$

Applying Theorem 3.1 on the functions $\mathfrak{L}$ and $f^{(n)}$ we obtain (3.8).

The integral version of the above theorem can be stated as:

Theorem 3.4. Let $-\infty<\alpha<\beta<\infty$ and $\alpha \leq a_{1}<a_{2} \cdots<a_{r} \leq \beta,(r \geq 2)$ be the given points, and $f \in C^{n}([\alpha, \beta])$ such that $(\cdot-\alpha)(\beta-\cdot)\left[f^{(n+1)}\right]^{2} \in L[\alpha, \beta]$ and $x, y:[a, b] \rightarrow[\alpha, \beta], w:[a, b] \rightarrow \mathbb{R}$ be continuous functions. Also let $H_{i j}$ be the fundamental polynomials of the Hermite basis and the functions $G_{c}(c=1,2,3,4)$ and $\mathfrak{J}$ be defined by (2.2)-(2.5) and (3.4) respectively. Then

$$
\begin{array}{r}
\int_{a}^{b} w(\tau) f(x(\tau)) d \tau-\int_{a}^{b} w(\tau) f(y(\tau)) d \tau=\left(\int_{a}^{b} w(\tau) x(\tau) d \tau-\int_{a}^{b} w(\tau) y(\tau) d \tau\right) f^{\prime}(\alpha) \\
+\int_{\alpha}^{\beta}\left[\int_{a}^{b} w(\tau)\left(G_{c}(x(\tau), t)-G_{c}(y(\tau), t)\right) d \tau\right] \sum_{j=1}^{r} \sum_{i=0}^{k_{j}} f^{(i+2)}\left(a_{j}\right) H_{i j}(t) d t \\
+\frac{f^{(n-1)}(\beta)-f^{(n-1)}(\alpha)}{\beta-\alpha} \int_{\alpha}^{\beta} \mathfrak{J}(s) d s+\mathbb{R} \tilde{\mathbb{E}} \mathbb{M}(f ; \alpha, \beta),
\end{array}
$$

where the remainder $\mathbb{R} \tilde{\mathbb{E}} \mathbb{M}(f ; \alpha, \beta)$ satisfies the estimation

$$
|\mathbb{R} \tilde{\mathbb{E}} \mathbb{M}(f ; \alpha, \beta)| \leq \frac{\sqrt{\beta-\alpha}}{\sqrt{2}}[\Lambda(\mathfrak{J}, \mathfrak{J})]^{\frac{1}{2}}\left|\int_{\alpha}^{\beta}(s-\alpha)(\beta-s)\left[f^{(n+1)}(s)\right]^{2} d s\right|^{\frac{1}{2}} .
$$

Using Theorem 3.2 we obtain the following Grüss type inequalities.

Theorem 3.5. Let $-\infty<\alpha<\beta<\infty$ and $\alpha \leq a_{1}<a_{2} \cdots<a_{r} \leq \beta,(r \geq 2)$ be the given points, and $f \in C^{n}([\alpha, \beta])$ such that $f^{(n)}$ is monotonic non decreasing on $[\alpha, \beta]$ and let $\mathfrak{L}$ be defined by (3.3). Then the 
representation (3.7) holds and the remainder $\mathbb{R} \mathbb{E M}(f ; \alpha, \beta)$ satisfies the bound

$$
|\mathbb{R} \mathbb{E M}(f ; \alpha, \beta)| \leq\left\|\mathfrak{L}^{\prime}\right\|_{\infty}\left\{\frac{f^{(n-1)}(\beta)+f^{(n-1)}(\alpha)}{2}-\frac{f^{(n-2)}(\beta)-f^{(n-2)}(\alpha)}{\beta-\alpha}\right\} .
$$

Proof. Since $\mathbb{R E M}(f ; \alpha, \beta)=(\beta-\alpha) \Lambda\left(\mathfrak{L}, f^{(n)}\right)$, applying Theorem 3.2 on the functions $\mathfrak{L}$ and $f^{(n)}$ we get $(3.11)$.

Integral case of the above theorem can be given:

Theorem 3.6. Let $-\infty<\alpha<\beta<\infty$ and $\alpha \leq a_{1}<a_{2} \cdots<a_{r} \leq \beta,(r \geq 2)$ be the given points, and $f \in C^{n}([\alpha, \beta])$ such that $f^{(n)}$ is monotonic non decreasing on $[\alpha, \beta]$ and let $x, y:[a, b] \rightarrow[\alpha, \beta], w:[a, b] \rightarrow \mathbb{R}$ be continuous functions and also $G_{c}(c=1,2,3,4)$ and $\mathfrak{J}$ be defined by (2.2)-(2.5) and(3.4) respectively. Then we have the representation (3.9) and the remainder $\mathbb{R} \tilde{\mathbb{E}} \mathbb{M}(f ; \alpha, \beta)$ satisfies the bound

$$
|\mathbb{R} \tilde{\mathbb{E}} \mathbb{M}(f ; \alpha, \beta)| \leq\left\|\mathfrak{J}^{\prime}\right\|_{\infty}\left\{\frac{f^{(n-1)}(\beta)+f^{(n-1)}(\alpha)}{2}-\frac{f^{(n-2)}(\beta)-f^{(n-2)}(\alpha)}{\beta-\alpha}\right\} .
$$

We present the Ostrowski-type inequalities related to generalizations of majorization inequality.

Theorem 3.7. Suppose that all assumptions of Theorem 2.2 hold. Assume (u,v) is a pair of conjugate exponents, that is $1 \leq u, v \leq \infty, 1 / u+1 / v=1$. Let $\left|f^{(n)}\right|^{u}:[\alpha, \beta] \rightarrow \mathbb{R}$ be an R-integrable function for some $n \in \mathbb{N}$. Then we have:

$$
\begin{aligned}
\mid \sum_{l=1}^{m} w_{l} f\left(x_{l}\right)- & \sum_{l=1}^{m} w_{l} f\left(y_{l}\right)-\left(\sum_{l=1}^{m} w_{l} x_{l}-\sum_{l=1}^{m} w_{l} y_{l}\right) f^{\prime}(\alpha) \\
& -\int_{\alpha}^{\beta}\left[\sum_{l=1}^{m} w_{l}\left(G_{c}\left(x_{l}, t\right)-G_{c}\left(y_{l}, t\right)\right)\right] \sum_{j=1}^{r} \sum_{i=0}^{k_{j}} f^{(i+2)}\left(a_{j}\right) H_{i j}(t) d t \mid \leq\left\|f^{(n)}\right\|_{u}\|\mathfrak{L}\|_{v},
\end{aligned}
$$

where $\mathfrak{L}$ is defined in (3.3).

The constant on the right-hand side of (3.13) is sharp for $1<u \leq \infty$ and the best possible for $u=1$.

Proof. By using (3.3) we have

$$
\mathfrak{L}(t)=\int_{\alpha}^{\beta}\left[\sum_{l=1}^{m} w_{l}\left(G_{c}\left(x_{l}, t\right)-G_{c}\left(y_{l}, t\right)\right)\right] G_{H, n-2}(t, s) d t, \text { for } c=1,2,3,4 .
$$

Using the identity (2.13) and applying Hölder's inequality we obtain

$$
\begin{aligned}
& \mid \sum_{l=1}^{m} w_{l} f\left(x_{l}\right)-\sum_{l=1}^{m} w_{l} f\left(y_{l}\right) \\
& -\left(\sum_{l=1}^{m} w_{l} x_{l}-\sum_{l=1}^{m} w_{l} y_{l}\right) f^{\prime}(\alpha)-\int_{\alpha}^{\beta}\left[\sum_{l=1}^{m} w_{l}\left(G_{c}\left(x_{l}, t\right)-G_{c}\left(y_{l}, t\right)\right)\right] \sum_{j=1}^{r} \sum_{i=0}^{k_{j}} f^{(i+2)}\left(a_{j}\right) H_{i j}(t) d t \mid \\
& =\left|\int_{\alpha}^{\beta} \mathfrak{L}(t) f^{(n)}(t) d t\right| \leq\left.\left\|f^{(n)}\right\|\right|_{u}\left(\int_{\alpha}^{\beta}|\mathfrak{L}(t)|^{v} d t\right)^{\frac{1}{v}} .
\end{aligned}
$$


For the proof of the sharpness of the constant $\left(\int_{\alpha}^{\beta}|\mathfrak{L}(t)|^{v} d t\right)^{\frac{1}{v}}$ is analog to one in proof of Theorem 11 in $[1]$.

Integral version of the above theorem can be given as:

Theorem 3.8. Suppose that all assumptions of Theorem 2.3 hold. Assume (u,v) is a pair of conjugate exponents, that is $1 \leq u, v \leq \infty, 1 / u+1 / v=1$. Let $\left|f^{(n)}\right|^{u}:[\alpha, \beta] \rightarrow \mathbb{R}$ be an R-integrable function for some $n \in \mathbb{N}$. Then we have:

$$
\begin{gathered}
\mid \int_{a}^{b} w(\tau) f(x(\tau)) d \tau-\int_{a}^{b} w(\tau) f(y(\tau)) d \tau-\left(\int_{a}^{b} w(\tau)(x(\tau)-y(\tau)) d \tau\right) f^{\prime}(\alpha) \\
-\int_{\alpha}^{\beta}\left[\int_{a}^{b} w(\tau)\left(G_{c}(x(\tau), t)-G_{c}(y(\tau), t)\right) d \tau\right] \sum_{j=1}^{r} \sum_{i=0}^{k_{j}} f^{(i+2)}\left(a_{j}\right) H_{i j}(t) d t \mid \\
\leq\left\|f^{(n)}\right\|_{u}\|\mathfrak{J}\|_{v}
\end{gathered}
$$

where $\mathfrak{J}$ is defined in (3.4).

The constant on the right-hand side of (3.14) is sharp for $1<u \leq \infty$ and the best possible for $u=1$.

\section{4. $n$-EXPONENTIAL CONVEXITY AND EXPONENTIAL CONVEXITY}

We begin this section by giving some definitions and notions which are used frequently in the results. For more details see e.g. [5], [9] and [14].

Definition 4.1. A function $f: I \rightarrow \mathbb{R}$ is $n$-exponentially convex in the Jensen sense on $I$ if

$$
\sum_{i, j=1}^{n} \xi_{i} \xi_{j} f\left(\frac{x_{i}+x_{j}}{2}\right) \geq 0
$$

hold for all choices $\xi_{1}, \ldots, \xi_{n} \in \mathbb{R}$ and all choices $x_{1}, \ldots, x_{n} \in$ I. A function $f: I \rightarrow \mathbb{R}$ is $n$-exponentially convex if it is n-exponentially convex in the Jensen sense and continuous on $I$.

Definition 4.2. A function $f: I \rightarrow \mathbb{R}$ is exponentially convex in the Jensen sense on $I$ if it is $n$-exponentially convex in the Jensen sense for all $n \in \mathbb{N}$.

A function $f: I \rightarrow \mathbb{R}$ is exponentially convex if it is exponentially convex in the Jensen sense and continuous.

Proposition 4.1. If $f: I \rightarrow \mathbb{R}$ is an $n$-exponentially convex in the Jensen sense, then the matrix $\left[f\left(\frac{x_{i}+x_{j}}{2}\right)\right]_{i, j=1}^{m}$ is a positive semi-definite matrix for all $m \in \mathbb{N}, m \leq n$. Particularly,

$$
\operatorname{det}\left[f\left(\frac{x_{i}+x_{j}}{2}\right)\right]_{i, j=1}^{m} \geq 0,
$$

for all $m \in \mathbb{N}, m=1,2, \ldots, n$. 
Remark 4.1. It is known that $f: I \rightarrow \mathbb{R}^{+}$is a log-convex in the Jensen sense if and only if

$$
\alpha^{2} f(x)+2 \alpha \beta f\left(\frac{x+y}{2}\right)+\beta^{2} f(y) \geq 0,
$$

holds for every $\alpha, \beta \in \mathbb{R}$ and $x, y \in I$. It follows that a positive function is log-convex in the Jensen sense if and only if it is 2-exponentially convex in the Jensen sense.

A positive function is log-convex if and only if it is 2-exponentially convex.

Motivated by inequalities (2.19) and (2.21), under the assumptions of Theorems 2.4 and 2.5 we define the following linear functionals:

$$
\begin{aligned}
\mathbb{H}_{1}(f)=\sum_{l=1}^{m} w_{l} f\left(x_{l}\right)-\sum_{l=1}^{m} w_{l} f\left(y_{l}\right)-\left(\sum_{l=1}^{m} w_{l} x_{l}-\sum_{l=1}^{m} w_{l} y_{l}\right) f^{\prime}(\alpha) \\
\quad-\int_{\alpha}^{\beta}\left[\sum_{l=1}^{m} w_{l}\left(G_{c}\left(x_{l}, t\right)-G_{c}\left(y_{l}, t\right)\right)\right] \sum_{j=1}^{r} \sum_{i=0}^{k_{j}} f^{(i+2)}\left(a_{j}\right) H_{i j}(t) d t, \quad c=1,2,3,4,
\end{aligned}
$$

and

$$
\begin{aligned}
& \mathbb{H}_{2}(f)=\int_{a}^{b} w(\tau) f(x(\tau)) d \tau-\int_{a}^{b} w(\tau) f(y(\tau)) d \tau-\left(\int_{a}^{b} w(\tau)(x(\tau)-y(\tau)) d \tau\right) f^{\prime}(\alpha) \\
& -\int_{\alpha}^{\beta}\left[\int_{a}^{b} w(\tau)\left(G_{c}(x(\tau), t)-G_{c}(y(\tau), t)\right) d \tau\right] \sum_{j=1}^{r} \sum_{i=0}^{k_{j}} f^{(i+2)}\left(a_{j}\right) H_{i j}(t) d t, \quad c=1,2,3,4 .
\end{aligned}
$$

Remark 4.2. Under the assumptions of Theorems 2.4 and 2.5 , it holds $\mathbb{H}_{i}(f) \geq 0, i=1,2$, for all $n$-convex functions $f$.

Lagrange and Cauchy type mean value theorems related to defined functionals are given in the following theorems.

Theorem 4.1. Let $f:[\alpha, \beta] \rightarrow \mathbb{R}$ be such that $f \in C^{n}[\alpha, \beta]$. If the inequalities in (2.18) (i=1) and (2.20) $(i=2)$ hold, then there exist $\xi_{i} \in[\alpha, \beta]$ such that

$$
\mathbb{H}_{i}(f)=f^{(n)}\left(\xi_{i}\right) \mathbb{H}_{i}(\varphi), \quad i=1,2,
$$

where $\varphi(x)=\frac{x^{n}}{n !}$ and $\mathbb{H}_{i}, i=1,2$ are defined by (4.1) and(4.2).

Proof. Similar to the proof of Theorem 4.1 in [10].

Theorem 4.2. Let $f, g:[\alpha, \beta] \rightarrow \mathbb{R}$ be such that $f, g \in C^{n}[\alpha, \beta]$. If the inequalities in (2.18) (i=1), (2.20) $(i=2)$, hold, then there exist $\xi_{i} \in[\alpha, \beta]$ such that

$$
\frac{\mathbb{H}_{i}(f)}{\mathbb{H}_{i}(g)}=\frac{f^{(n)}\left(\xi_{i}\right)}{g^{(n)}\left(\xi_{i}\right)}, \quad i=1,2,
$$

provided that the denominators are non-zero and $\mathbb{H}_{i}, i=1,2$, are defined by (4.1) and(4.2). 
Proof. Similar to the proof of Theorem 4.2 in [10].

Now we will produce $n$-exponentially and exponentially convex functions applying defined functionals. We use an idea from [14]. In the sequel $J$ will be interval in $\mathbb{R}$.

Theorem 4.3. Let $\Omega=\left\{f_{t}: t \in J\right\}$, where $J$ is an interval in $\mathbb{R}$, be a family of functions defined on an interval $[\alpha, \beta]$ such that the function $t \mapsto\left[x_{0}, \ldots, x_{n} ; f_{t}\right]$ is $n$-exponentially convex in the Jensen sense on $J$ for every $(n+1)$ mutually different points $x_{0}, \ldots, x_{n} \in[\alpha, \beta]$. Then for the linear functionals $\mathbb{H}_{i}\left(f_{t}\right)$ $(i=1,2)$ as defined by (4.1) and (4.2), the following statements hold:

(i) The function $t \rightarrow \mathbb{H}_{i}\left(f_{t}\right)$ is n-exponentially convex in the Jensen sense on $J$ and the matrix $\left[\mathbb{H}_{i}\left(f_{\frac{t_{j}+t_{l}}{2}}\right)\right]_{j, l=1}^{m}$ is a positive semi-definite for all $m \in \mathbb{N}, m \leq n, t_{1}, . ., t_{m} \in J$. Particularly,

$$
\operatorname{det}\left[\mathbb{H}_{i}\left(f_{\frac{t_{j}+t_{l}}{2}}\right)\right]_{j, l=1}^{m} \geq 0 \text { for all } m \in \mathbb{N}, m=1,2, \ldots, n \text {. }
$$

(ii) If the function $t \rightarrow \mathbb{H}_{i}\left(f_{t}\right)$ is continuous on $J$, then it is n-exponentially convex on $J$.

Proof. The proof is similar to the proof of Theorem 23 in [2].

The following corollary is an immediate consequence of the above theorem.

Corollary 4.1. Let $\Omega=\left\{f_{t}: t \in J\right\}$, where $J$ is an interval in $\mathbb{R}$, be a family of functions defined on an interval $[\alpha, \beta]$ such that the function $t \mapsto\left[x_{0}, \ldots, x_{n} ; f_{t}\right]$ is exponentially convex in the Jensen sense on $J$ for every $(n+1)$ mutually different points $x_{0}, \ldots, x_{n} \in[\alpha, \beta]$. Then for the linear functionals $\mathbb{H}_{i}\left(f_{t}\right)(i=1,2)$ as defined by (4.1) and (4.2), the following statements hold:

(i) The function $t \rightarrow \mathbb{H}_{i}\left(f_{t}\right)$ is exponentially convex in the Jensen sense on $J$ and the matrix $\left[\mathbb{H}_{i}\left(f_{\frac{f_{j}+t_{l}}{2}}\right)\right]_{j, l=1}^{m}$ is a positive semi-definite for all $m \in \mathbb{N}, m \leq n, t_{1}, . ., t_{m} \in J$. Particularly,

$$
\operatorname{det}\left[\mathbb{H}_{i}\left(f_{\frac{t_{j}+t_{l}}{2}}\right)\right]_{j, l=1}^{m} \geq 0 \text { for all } m \in \mathbb{N}, m=1,2, \ldots, n .
$$

(ii) If the function $t \rightarrow \mathbb{H}_{i}\left(f_{t}\right)$ is continuous on $J$, then it is exponentially convex on $J$.

Corollary 4.2. Let $\Omega=\left\{f_{t}: t \in J\right\}$, where $J$ is an interval in $\mathbb{R}$, be a family of functions defined on an interval $[\alpha, \beta]$ such that the function $t \mapsto\left[x_{0}, \ldots, x_{n} ; f_{t}\right]$ is 2-exponentially convex in the Jensen sense on $J$ for every $(n+1)$ mutually different points $x_{0}, \ldots, x_{n} \in[\alpha, \beta]$. Let $\mathbb{H}_{i}, i=1,2$ be linear functionals defined by (4.1) and (4.2). Then the following statements hold:

(i) If the function $t \mapsto \mathbb{H}_{i}\left(f_{t}\right)$ is continuous on $J$, then it is 2-exponentially convex function on $J$. If $t \mapsto \mathbb{H}_{i}\left(f_{t}\right)$ is additionally strictly positive, then it is also log-convex on J. Furthermore, the following inequality holds true:

$$
\left[\mathbb{H}_{i}\left(f_{s}\right)\right]^{t-r} \leq\left[\mathbb{H}_{i}\left(f_{r}\right)\right]^{t-s}\left[\mathbb{H}_{i}\left(f_{t}\right)\right]^{s-r}, \quad i=1,2
$$


for every choice $r, s, t \in J$, such that $r<s<t$.

(ii) If the function $t \mapsto \mathbb{H}_{i}\left(f_{t}\right)$ is strictly positive and differentiable on $J$, then for every $p, q, u, v \in J$, such that $p \leq u$ and $q \leq v$, we have

$$
\mu_{p, q}\left(\mathbb{H}_{i}, \Omega\right) \leq \mu_{u, v}\left(\mathbb{H}_{i}, \Omega\right)
$$

where

$$
\mu_{p, q}\left(\mathbb{H}_{i}, \Omega\right)= \begin{cases}\left(\frac{\mathbb{H}_{i}\left(f_{p}\right)}{\mathbb{H}_{i}\left(f_{q}\right)}\right)^{\frac{1}{p-q}}, & p \neq q \\ \exp \left(\frac{\frac{d}{d p} \mathbb{H}_{i}\left(f_{p}\right)}{\mathbb{H}_{i}\left(f_{p}\right)}\right), & p=q\end{cases}
$$

for $f_{p}, f_{q} \in \Omega$.

Proof. The proof is similar to the proof of Corollary 2 in [2].

Remark 4.3. Note that the results from Theorem 4.3, Corollary 4.1 and Corollary 4.2 still hold when two of the points $x_{0}, \ldots, x_{l} \in[\alpha, \beta]$ coincide, say $x_{1}=x_{0}$, for a family of differentiable functions $f_{t}$ such that the function $t \mapsto\left[x_{0}, \ldots, x_{l} ; f_{t}\right]$ is an n-exponentially convex in the Jensen sense (exponentially convex in the Jensen sense, log-convex in the Jensen sense), and furthermore, they still hold when all $(l+1)$ points coincide for a family of l differentiable functions with the same property. The proofs are obtained by suitable characterization of convexity.

\section{Applications}

In this section, we give some applications of our generalized results about the upper bounds as well as exponential convex functions.

Firstly, we consider some related analytical inequalities by using our generalized results of upper bounds.

Example 5.1. By using Ostrowski-type inequality (3.13) for $n=4$ as an upper bound of our generalized results,

- let $f(x)=e^{x}, x \in \mathbb{R}$, then

$$
0 \leq\left|\sum_{l=1}^{m} w_{l} e^{x_{l}}-\sum_{l=1}^{m} w_{l} e^{y_{l}}-\left(\sum_{l=1}^{m} w_{l} x_{l}-\sum_{l=1}^{m} w_{l} y_{l}\right) e^{\alpha}-\mathbb{G H}_{c}\right| \leq \frac{\left(e^{u \beta}-e^{u \alpha}\right)^{\frac{1}{u}}}{u^{\frac{1}{u}}}\|\mathfrak{L}\|_{v},
$$

- let $f(x)=x^{r},[0, \infty)$ for $r>3$, then

$$
\begin{aligned}
0 \leq & \left|\sum_{l=1}^{m} w_{l} x_{l}^{r}-\left(\sum_{l=1}^{m} w_{l} x_{l}-\sum_{l=1}^{m} w_{l} y_{l}\right) r \alpha^{r-1}-\mathbb{G H}_{c}\right| \\
& \leq \frac{r(r-1)(r-2)(r-3)}{(u(r-4)+1)^{\frac{1}{u}}}\left(\beta^{u(r-4)+1}-\alpha^{u(r-4)+1}\right)^{\frac{1}{u}}\|\mathfrak{L}\|_{v},
\end{aligned}
$$


- let $f(x)=x \log x, x \in(0, \infty)$, then

$$
\begin{aligned}
0 \leq & \sum_{l=1}^{m} w_{l} x_{l} \log x_{l}-\sum_{l=1}^{m} w_{l} y_{l} \log y_{l}-\left(\sum_{l=1}^{m} w_{l} x_{l}-\sum_{l=1}^{m} w_{l} y_{l}\right)(\log \alpha+1)-\mathbb{G H}_{c} \mid \\
& \leq \frac{2}{(1-3 u)^{\frac{1}{u}}}\left(\beta^{1-3 u}-\alpha^{1-3 u}\right)^{\frac{1}{u}}\|\mathfrak{L}\|_{v},
\end{aligned}
$$

- let $f(x)=-\log x, x \in(0, \infty)$, then

$$
\begin{aligned}
0 \leq & \left|\sum_{l=1}^{m} w_{l} \log y_{l}-\sum_{l=1}^{m} w_{l} \log x_{l}+\left(\sum_{l=1}^{m} w_{l} x_{l}-\sum_{l=1}^{m} w_{l} y_{l}\right) \frac{1}{\alpha}-\mathbb{G H}_{c}\right| \\
& \leq \frac{6}{(1-4 u)^{\frac{1}{u}}}\left(\beta^{1-4 u}-\alpha^{1-4 u}\right)^{\frac{1}{u}}\|\mathfrak{L}\|_{v}
\end{aligned}
$$

where, $\mathbb{G H}_{c}=\int_{\alpha}^{\beta}\left[\sum_{l=1}^{m} w_{l}\left(G_{c}\left(x_{l}, t\right)-G_{c}\left(y_{l}, t\right)\right)\right] \sum_{j=1}^{r} \sum_{i=0}^{k_{j}} f^{(i+2)}\left(a_{j}\right) H_{i j}(t) d t, \quad(c=1,2,3,4)$, and $\mathfrak{L}(s)=$ $\int_{\alpha}^{\beta}\left[\sum_{l=1}^{m} w_{l}\left(G_{c}\left(x_{l}, t\right)-G_{c}\left(y_{l}, t\right)\right)\right] G_{H, 2}(t, s) d t$.

We can also give the particular cases of above results for $u=1$ and $v=\infty$.

Now, we construct exponentially convex function by using family of convex functions defined on $(0, \infty)$ :

Example 5.2. Let

$$
\mathbb{E}_{1}=\left\{\theta_{v}:(0, \infty) \rightarrow(0, \infty): v \in \mathbb{R}\right\}
$$

be a family of continuous convex functions defined by

$$
\theta_{v}(x)= \begin{cases}\frac{x e^{v x}}{v^{2}}, & v \neq 0 \\ \frac{x^{3}}{2}, & v=0 .\end{cases}
$$

We have $v \mapsto\left(\frac{\theta_{v}(x)}{x}\right)^{\prime \prime}(t \in \mathbb{R})$ is exponentially convex for every fixed $x \in \mathbb{R}$. Using analogous arguing as in the proof of Theorem 4.3 we also have that $v \mapsto \theta_{v}\left[z_{0}, \ldots, z_{t}\right]$ is exponentially convex (and so exponentially convex in the Jensen sense). Using Corollary 4.1 we conclude that $v \mapsto \mathbb{H}_{i}\left(\theta_{v}\right)$ is exponentially convex in the Jensen sense. It is easy to verify that this mapping is continuous (although mapping $v \mapsto \theta_{v}$ is not continuous for $v=0$ ), so it is exponentially convex.

For this family of functions, $\mu_{v, q}\left(\mathbb{E}_{1}, \mathbb{H}_{i}\right)$ from (4.6), becomes

$$
\mu_{p, q}\left(\mathbb{H}_{i}, \mathbb{E}_{1}\right)= \begin{cases}\left(\frac{\mathbb{H}_{i}\left(\theta_{p}\right)}{\mathbb{H}_{i}\left(\theta_{q}\right)}\right)^{\frac{1}{p-q}}, & p \neq q, \\ \exp \left(\frac{\mathbb{H}_{i}\left(i d \cdot \theta_{p}\right)}{\mathbb{H}_{i}\left(\theta_{p}\right)}-\frac{n}{p}\right), & p=q \neq 0, \\ \exp \left(\frac{1}{n+1} \frac{\mathbb{H}_{i}\left(i d \cdot \phi_{0}\right)}{\mathbb{H}_{i}\left(\theta_{0}\right)}\right), & p=q=0,\end{cases}
$$

where id is the identity function. 
Now using (4.5), $\mu_{p, q}$ is monotone function in parameters $p$ and $q$.

We observe here that $\left(\frac{\frac{d^{2} \theta_{p}}{\frac{d x^{2}}{d^{2} \theta_{q}}}}{d x^{2}}\right)^{\frac{1}{p-q}}(\ln x)=x$ so using Theorem 4.2 it follows that

$$
M_{p, q}\left(\mathbb{E}_{1}, \mathbb{H}_{i}\right)=\ln \mu_{p, q}\left(\mathbb{E}_{1}, \mathbb{H}_{i}\right)
$$

satisfies

$$
\alpha \leq M_{p, q}\left(\mathbb{E}_{1}, \mathbb{H}_{i}\right) \leq \beta, \quad i=1,2
$$

This shows that $M_{p, q}\left(\mathbb{E}_{1}, \mathbb{H}_{i}\right)$ is mean. Because of the above inequality (4.5), this mean is also monotonic.

Remark 5.1. We can construct other examples for exponentially convex functions as Example 2 for the families of continuous convex functions:

\section{$\bullet$}

$$
\mathbb{E}_{2}=\left\{\mu_{t}:(0, \infty) \rightarrow \mathbb{R}: t \in \mathbb{R}\right\}
$$

where,

$$
\mu_{t}(x)= \begin{cases}\frac{x^{t+1}}{t(t-1)}, & t \neq 0,1 \\ -x \log x, & t=0 \\ x^{2} \log x, & t=1\end{cases}
$$

$\bullet$

$$
\mathbb{E}_{3}=\left\{\chi_{t}:(0, \infty) \rightarrow(0, \infty): t \in(0, \infty)\right\}
$$

where,

$$
\chi_{t}(x)= \begin{cases}\frac{x t^{-x}}{\log ^{2} t}, & t \neq 1 \\ \frac{x^{3}}{2}, & t=1\end{cases}
$$

$$
\mathbb{E}_{4}=\left\{\delta_{t}:(0, \infty) \rightarrow(0, \infty): t \in(0, \infty)\right\}
$$

where,

$$
\delta_{t}(x):=\frac{x e^{-x \sqrt{t}}}{t}
$$

\section{Conflict of Interests:}

The authors declare that there is no conflict of interests.

\section{Acknowledgment:}

The publication was supported by the Ministry of Education and Science of the Russian Federation (the 
Agreement number No. 02.a03.21.0008.) This publication is partially supported by Royal Commission for Jubail and Yanbu, Kingdom of Saudi Arabia.

\section{REFERENCES}

[1] R. P. Agarwal, S. Ivelić Bradanović and J. Pečarić, Generalizations of Sherman's inequality by Lidstone's interpolating polynomial, J. Inequal. Appl., 2016 (2016), Art. ID 6.

[2] M. Adil Khan, N. Latif and J. Pečarić, Generalization of majorization theorem, J. Math. Inequal., 9(3) (2015), 847-872.

[3] R. P. Agarwal and P. J. Y. Wong, Error Inequalities in Polynomial Interpolation and Their Applications, Kluwer Academic Publishers, Dordrecht/ Boston/ London, 1993.

[4] P. R. Beesack, On the Greens function of an N-point boundary value problem, Pacific J. Math. 12 (1962), 801-812. Kluwer Academic Publishers, Dordrecht / Boston / London, 1993.

[5] S. N. Bernstein, Sur les fonctions absolument monotones, Acta Math. 52 (1929), 1-66.

[6] P. Cerone and S. S. Dragomir, Some new Ostrowski-type bounds for the Čebyšev functional and applications, J. Math. Inequal. 8(1) (2014), 159-170.

[7] P. J. Davis, Interpolation and Approximation, Blaisedell Publishing Co., Boston, 1961.

[8] L. Fuchs, A new proof of an inequality of Hardy-Littlewood-Polya, Mat. Tidsskr, B (1947), 53-54.

[9] J. Jakšetić and J. Pečarić, Exponential convexity method, J. Convex Anal. 20(2013), no. 1, 181-197.

[10] J. Jakšetić, J. Pečarić and A. Perušić, Steffensen inequality, higher order convexity and exponential convexity, Rend. Circ. Mat. Palermo 63 (1) (2014), 109-127.

[11] N. Mahmood, R. P. Agarwal, S. I. Butt and J. Pečarić, New Generalization of Popoviciu type inequalities via new Green functions and Montgomery identity, J. Inequal. Appl., 2017 (2017), Art. ID 108.

[12] A. W. Marshall, I. Olkin and Barry C. Arnold, Inequalities: Theory of Majorization and Its Applications (Second Edition), Springer Series in Statistics, New York 2011.

[13] J. Pečarić, F. Proschan and Y. L. Tong, Convex functions, Partial Orderings and Statistical Applications, Academic Press, New York, 1992.

[14] J. Pečarić and J. Perić, Improvements of the Giaccardi and the Petrović inequality and related results, An. Univ. Craiova Ser. Mat. Inform., 39(1) (2012), 65-75.

[15] J. Pečarić, On some inequalities for functions with nondecreasing increments, J. Math. Anal. Appl., 98 (1984), $188-197$.

[16] A. Yu. Levin, Some problems bearing on the oscillation of solutions of linear differential equations, Soviet Math. Dokl., 4(1963), 121-124. 